\title{
Novel antenna concepts via coordinate transformation
}

\author{
Paul-Henri Tichit, Shah Nawaz Burokur, Xinying Wu, Dylan Germain, André de Lustrac* \\ IEF, Univ. Paris-Sud, CNRS, UMR 8622, 91405 Orsay cedex, France
*corresponding author, E-mail: andre.de-lustrac@u-psud.fr
}

\begin{abstract}
Coordinate transformation is an emerging field which offers a powerful and unprecedented ability to manipulate and control electromagnetic waves. Using this tool, we demonstrate the design of novel antenna concepts by tailoring their radiation properties. The wave manipulation is enabled through the use of engineered dispersive composite metamaterials that realize the space coordinate transformation. Three types of antennas are considered for design: a directive, a beam steerable and a quasi-isotropic one. Numerical simulations together with experimental measurements are performed in order to validate the coordinate transformation concept. Near-field cartography and far-field pattern measurements performed on a fabricated prototype agree qualitatively with Finite Element Method (FEM) simulations. It is shown that a particular radiation pattern can be tailored at ease into a desired one by modifying the electromagnetic properties of the space around radiator. This idea opens the way to novel antenna design techniques for various application domains such as the aeronautical and transport fields.
\end{abstract}

\section{Introduction}

Coordinate transformation (also called transformation optics or transformation electromagnetics) is a powerful mathematical tool that is used to generate a new transformed space from an initial one where solutions of Maxwell's equations are known by manipulating electromagnetic waves. As a first step, it consists in imagining a virtual space with desired topological properties, which will contain the underlying physics. This approach has been revived when J. B. Pendry et al. [1] have proposed an interpretation where permeability and permittivity tensors components can be viewed as a material in the original space. It is as if the new material mimicks the defined topological space. Since this pioneering work of J. B. Pendry and that of U. Leonhardt et al. [2], transformation optics is an emerging field where Maxwell's equations are form invariant under a coordinate transformation. It offers an unconventional strategy to the design of novel class metamaterial devices. The most striking application conceived so far via coordinate transformation concept is the invisibility cloak for which various designs have been presented in microwave [3-5] and optical regimes [6-8]. Other interesting wave manipulation applications such as wave concentrators [9], field rotators [10], electromagnetic wormholes [11], waveguide transitions and bends [12-16] have also been proposed. Concerning antenna applications, focusing lens antennas [17-19] and the engineering of radiation patterns [20] have been proposed. The performances of an omnidirectional retroreflector [21] based on the transmutation of singularities [22] and Luneberg lenses [23] have also been experimentally demonstrated. An octave-bandwidth horn antenna has experimentally validated for satellite communications [24]. Recently, techniques of source transformation [25-27] have offered new opportunities for the design of active devices with source distribution included in the transformed space.

Using this last approach, we review the design of three antenna models where the radiation pattern is tailored specifically in each case. The first one concerns an ultradirective antenna obtained by stretching a source into an extended coherent radiator [28-30]. The design has been implemented through the use of judiciously engineered metamaterials and the device is shown experimentally to produce an ultra-directive emission. The idea has been extended to a second device, a wave bending one, so as to achieve a steered beam antenna via a rotational coordinate transformation. Experimental measurements have shown a beam steering as much as $66^{\circ}$. Finally, we present the numerical design of a quasi-isotropic antenna achieved by expanding the space around a directive source [31].

\section{Ultra-directive antenna}

The ultra-directive antenna is based on the transformation of a cylindrical space into a rectangular one. The schematic principle of the transformation is presented in Fig. 1. The theoretical underlying physics of the transformation involved here has been detailed recently in [28]. The concept is as follows: the imagined space of our proposed antenna is obtained by transforming a flat isotropic cylindrical half-space with zero Riemann curvature tensor described in polar coordinates $\{r, \theta\}$ into a flat space in squeezed Cartesian coordinates. $x^{\prime}, y^{\prime}$ and $z$ ' are the coordinates in the virtual transformed rectangular space and $\mathrm{x}, \mathrm{y}, \mathrm{z}$ are those in the initial real cylindrical space. We assume free space in the cylinder, with isotropic permeability and permittivity tensors $\varepsilon_{0}$ and $\mu_{0}$. In the theoretical study of [28], we have shown that the coordinate transformation can be implemented by a material obeying the following tensors: 


$$
\psi^{i^{\prime} j^{\prime}}=g^{i^{\prime} j^{\prime}}\left|\operatorname{det}\left(g^{i^{\prime} j^{\prime}}\right)\right|^{-\frac{1}{2}} \psi
$$

where $\psi$ represents the permittivity or permeability tensor and $g$, the metric tensor of our designed space. The material must then be able to produce the following dielectric tensors presenting no non-diagonal components (2):

$$
\varepsilon^{i j}=\mu^{i j}=\operatorname{diag}\left(\varepsilon_{x x}\left(x^{\prime}\right), \frac{1}{\varepsilon_{x x}\left(x^{\prime}\right)}, \alpha \varepsilon_{x x}\left(x^{\prime}\right)\right),
$$

where $\varepsilon_{x x}\left(x^{\prime}\right)=\frac{\pi x^{\prime}}{e}$ and $\alpha=\frac{d^{2}}{4 L^{2}}$, with $d$ representing the diameter of the initial cylindrical space and $e$ and $L$, respectively, the width and length of the rectangular target space. For a practical implementation using metamaterials, the dimensions of the semi-cylindrical space is set so that $\alpha$ $=4$ in order to obtain achievable values for the electromagnetic parameters. We also consider a polarized electromagnetic wave with an electric field pointing in the $z$-direction, which allows modifying the dispersion equation in order to simplify the electromagnetic parameters without changing Maxwell's equations and propagation in the structure. This leads to a metamaterial which is described with $e=0.15 \mathrm{~m}$ and $L=0.05 \mathrm{~m}$ by:

$$
\mu_{x x}=1 ; \mu_{y y}=\frac{1}{\left(\varepsilon_{x x}\right)^{2}} ; \varepsilon_{z z}=4\left(\varepsilon_{x x}\right)^{2},
$$
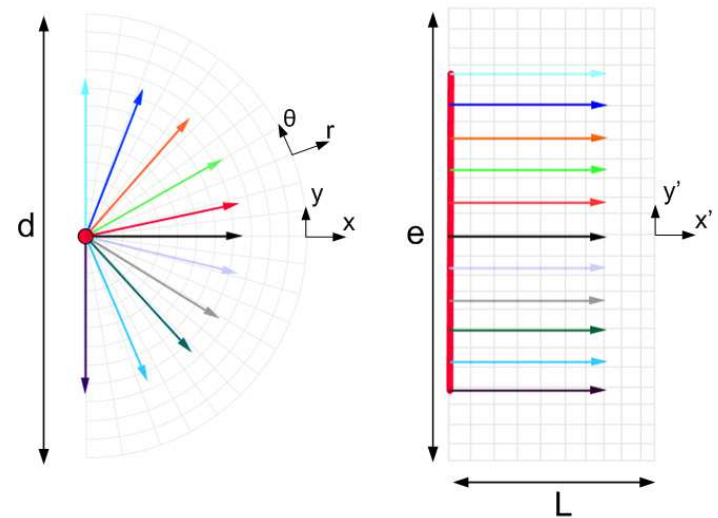

(a)

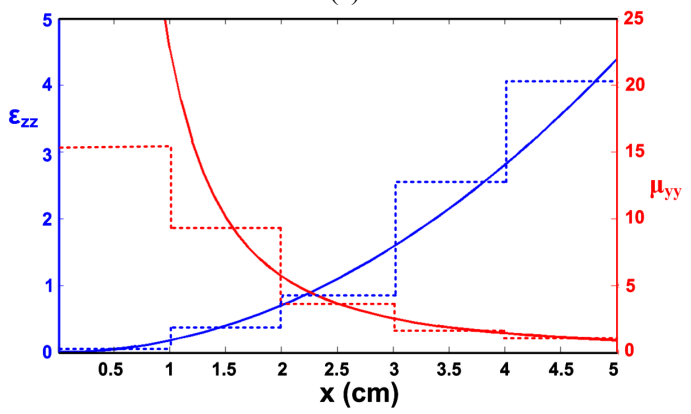

(b)

Figure 1: (a) Transformation of a cylindrical space into a rectangular one. (b) Continuous (continuous lines) and discretized (dashed lines) variations of permeability and permittivity of the material.
Discrete values are then created for the desired variation of $\mu_{\mathrm{yy}}$ and $\varepsilon_{\mathrm{zz}}$ to secure a practical realization producing experimental performances close to theory. Fig. 2 shows the photography of the fabricated prototype. A microstrip square patch antenna printed on a $0.787 \mathrm{~mm}$ thick low-loss dielectric substrate (Rogers RT/Duroid 5870TM with 17.5 $\mu \mathrm{m}$ copper cladding, $\varepsilon_{\mathrm{r}}=2.33$ and $\tan \delta=0.0012$ ) is used as radiating source. A surrounding material made of alternating electric metamaterial and magnetic metamaterial layers is used to capture the emanating omnidirectional radiation from the patch source and transform it into a directive one. The metamaterial is a discrete structure composed of five different regions where permittivity and permeability vary according to (3) and to the profile of Fig. 1(b).

The axial permittivity $\varepsilon_{\mathrm{zz}}$ and permeability $\mu_{\mathrm{yy}}$ show respectively values ranging from 0.12 to 4.15 and from 1.58 to 15.3 . The bulk metamaterial is assembled using 56 layers of dielectric boards on which subwavelength resonant structures are printed. 28 layers contain SRRs [32] and 28 others contain ELCs [33], shown in the insets of Fig. 2 and known to provide respectively a magnetic and an electric response. Each layer is made of 5 regions of metamaterials corresponding to the discretized values of Fig. 1(b). Because of constraints of the layout, we choose a rectangular unit cell with dimensions $3.333 \mathrm{~mm}$ for both resonators. The layout consists of 5 regions, each of which is three unit cells long $(10 \mathrm{~mm})$. We are able to obtain the desired $\varepsilon_{\mathrm{zz}}$ and $\mu_{\mathrm{yy}}$ by tuning the resonators' geometric parameters, as illustrated and detailed in Fig. 3. The SRR and ELC resonators are simulated with finite-element method based Ansys HFSS commercial code for the $[8 \mathrm{GHz}$ $-15 \mathrm{GHz}$ ] frequency band. The calculated $S$-parameters are then utilized to extract the effective material parameters $\mu_{\mathrm{yy}}$ and $\varepsilon_{\mathrm{zz}}$, through the use of a retrieval process described in [34]. $\mu_{\mathrm{yy}}$ and $\varepsilon_{\mathrm{zz}}$ are respectively shown in Fig. 4(a) and 4(b). The insets of Fig. 4 show the variation of the two material parameters in the vicinity of $10 \mathrm{GHz}$.

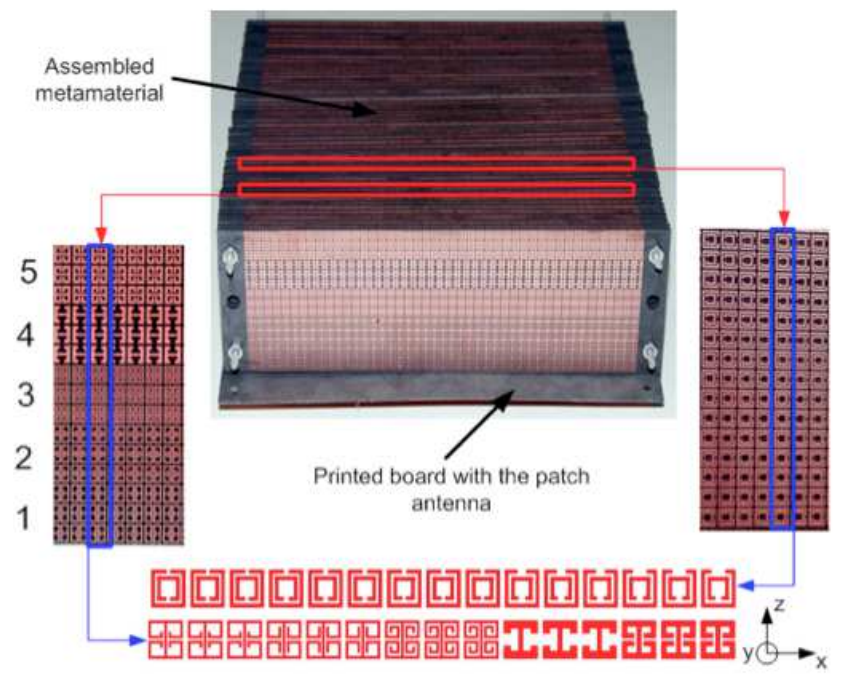

Figure 2: Photography of the structure of the antenna. The inserts show the permittivity (left) and the permeability (right) layers of the material. 
SRR

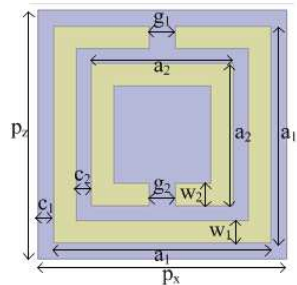

\begin{tabular}{|c|c|c|c|c|c|}
\hline region & $\mathrm{a}_{2}$ & $\mathrm{c}_{2}$ & $\mathrm{~g}_{1}$ & $\mathrm{~g}_{2}$ & $\mu_{\mathrm{yy}}$ \\
\hline 1 & 1.9 & 0.2 & 0.35 & 0.35 & 15.3 \\
\hline 2 & 1.9 & 0.2 & 0.38 & 0.38 & 10 \\
\hline 3 & 1.8 & 0.25 & 0.36 & 0.3 & 3.66 \\
\hline 4 & 1.8 & 0.25 & 0.8 & 0.6 & 1.89 \\
\hline 5 & 1.8 & 0.25 & 1.2 & 0.6 & 1.58 \\
\hline & $\mathrm{p}_{\mathrm{x}}=\mathrm{p}_{\mathrm{z}}=10 / 3, \mathrm{a}_{1}=2.9, \mathrm{w}_{1}=\mathrm{w}_{2}=0.3, \mathrm{c}_{1}=0.2165$
\end{tabular}

ELC

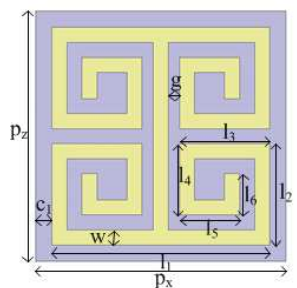

\begin{tabular}{|c|c|c|c|c|}
\hline region & $1_{4}$ & $1_{5}$ & $1_{6}$ & $\varepsilon_{\text {zz }}$ \\
\hline 1 & 0.5 & 0 & 0 & 0.15 \\
\hline 2 & 0.9 & 0 & 0 & 0.4 \\
\hline 3 & 0.95 & 0.8 & 0.55 & 0.65 \\
\hline
\end{tabular}

$\mathrm{p}_{\mathrm{x}}=\mathrm{p}_{\mathrm{z}}=10 / 3,1_{1}=2.9,1_{2}=1.35,1_{3}=1.2$,

$\mathrm{w}=0.2, \mathrm{~g}=0.15, \mathrm{c}_{1}=0.216$

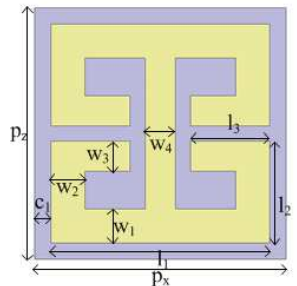

\begin{tabular}{|c|c|c|c|c|}
\hline region & $l_{2}$ & $l_{3}$ & $\mathrm{w}_{3}$ & $\varepsilon_{z z}$ \\
\hline 4 & 1 & 0 & 0 & 2.74 \\
\hline 5 & 1.35 & 1.05 & 0.4 & 4.15 \\
\hline
\end{tabular}

All dimensions are in $\mathrm{mm}$.

Figure 3: Unit cell of the SRR used as magnetic material and of the ELC used as electric material. The tables summarize the dimensions of these two metamaterial cells to achieve the different values of the electromagnetic parameters needed for the transformation.

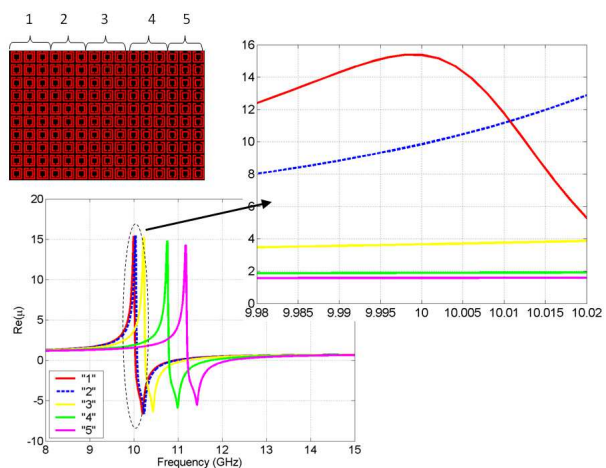

(a)

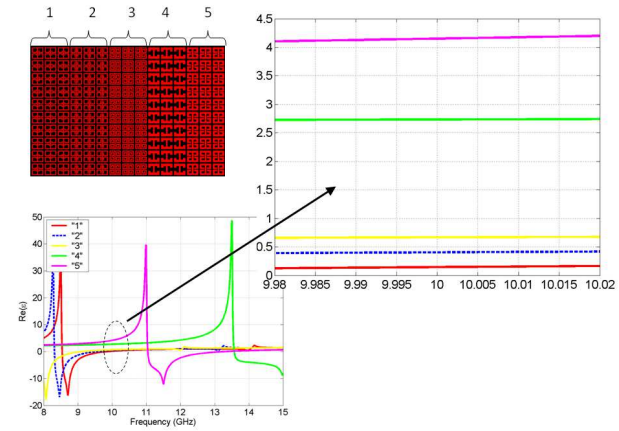

(b)

Figure 4: (a) Magnetic response of the SRR for the 5 regions. (b) Electric response of the ELC for the 5 regions.
The metamaterial layers are mounted 2 by 2 with a constant air spacing of $2.2 \mathrm{~mm}$ between each. Overall dimensions of the antenna are $15 \mathrm{~cm} \times 15 \mathrm{~cm} \times 5 \mathrm{~cm}$. Farfield patterns measurements are performed in an anechoic chamber, where the metamaterial-based antenna is used as emitter and a wideband $[2 \mathrm{GHz}-18 \mathrm{GHz}]$ is used as receiver. The E-plane radiation pattern is measured at 10.6 $\mathrm{GHz}$ for computer-controlled elevation angle varying from $90^{\circ}$ to $+90^{\circ}$. Fig. 5 presents the comparison between simulations and experiments for the patch source alone and the metamaterial antenna. The transformation of the patch's omnidirectional radiation into a directive is clearly established. A narrow half-power beamwidth of $13^{\circ}$ is observed for the measured antenna. These performances are competitive with classical high directivity antennas such as parabolic reflector antennas.

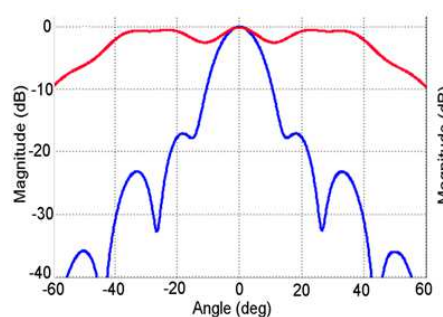

(a)

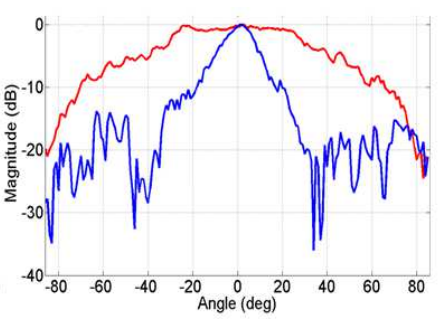

(b)
Figure 5: (a) Calculated and (b) measured radiation pattern at $10.6 \mathrm{GHz}$ of the proposed metamaterial antenna (blue) and the microstrip patch radiator alone (red).

\section{Azimuthal antenna}

Here, we propose a two-dimensional coordinate transformation, which transforms the vertical radiation of a plane source into a directive azimuthal emission. Let us consider a source radiating in a rectangular space. Theoretically this radiation emitted from the latter source can be transformed into an azimuthal one using transformation optics. The transformation procedure is noted $\mathrm{F}\left(\mathrm{x}^{\prime}, \mathrm{y}^{\prime}\right)$ and consists in bending the emission. Fig. 6 shows the operating principle of this rotational coordinate transformation. Mathematically, $\mathrm{F}\left(\mathrm{x}^{\prime}, \mathrm{y}^{\prime}\right)$ can be expressed as:

$$
\left\{\begin{array}{l}
x^{\prime}=\operatorname{axcos}(b y) \\
y^{\prime}=\operatorname{axsin}(b y) \\
z^{\prime}=z
\end{array}\right.
$$

where $x^{\prime}, y^{\prime}$, and $z^{\prime}$ are the coordinates in the bent space, and $\mathrm{x}, \mathrm{y}$ and $\mathrm{z}$ are those in the initial rectangular space. In the initial space, we assume free space. $\mathrm{L}_{2}-\mathrm{L}_{1}$ and $\mathrm{L}$ are respectively the width and the length of the rectangular space. The rotational transformation of Fig. 6 is defined by parameter $a$ considered as an "expansion" parameter and parameter $b$ which controls the rotation angle of the transformation $\mathrm{F}\left(\mathrm{x}^{\prime}, \mathrm{y}^{\prime}\right)$. By substituting the new coordinate system in the tensor components, and after some simplifications, the material parameters are derived. After 
diagonalization, calculations lead to permeability and permittivity tensors given in the diagonal base by:

$\bar{\varepsilon}=\left(\begin{array}{ccc}\psi_{\mathrm{rr}} & 0 & 0 \\ 0 & \psi_{\theta \theta} & 0 \\ 0 & 0 & \psi_{\mathrm{zz}}\end{array}\right) \varepsilon_{0} \quad \overline{\bar{\mu}}=\left(\begin{array}{ccc}\psi_{\mathrm{rr}} & 0 & 0 \\ 0 & \psi_{\theta \theta} & 0 \\ 0 & 0 & \psi_{\mathrm{zz}}\end{array}\right) \mu_{0}$

with: $\quad \psi_{r r}=\frac{a}{b r} ; \psi_{\theta \theta}=\frac{a}{b} r ; \psi_{z z}=\frac{1}{a b r}$,

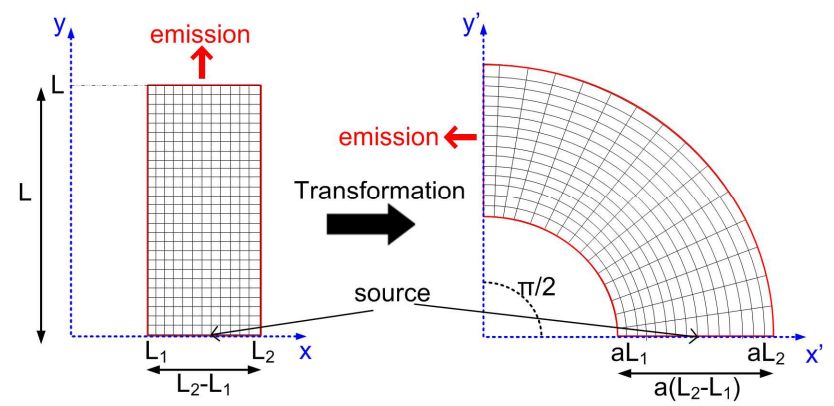

Figure 6: Schematic principle of the 2D rotational coordinate transformation. The emission in a rectangular space is transformed into an azimuthal one.

The transformation formulation is implemented using finite-element method based commercial solver Comsol Multiphysics. Fig. 7 shows the comparison of 2D simulation between a plane source made of current lines in $y z$ plane above a limited metallic ground plane (Fig. 7(a)) and the same source surrounded by a metamaterial defined by Eq. (5) [Fig. 7(b)]. Fig. 7(c) and 7(d) show respectively the far-field patterns of the plane source without and with the metamaterial structure. The left shift of the peak corresponds to a rotation of $76^{\circ}$ of the emitted radiation.

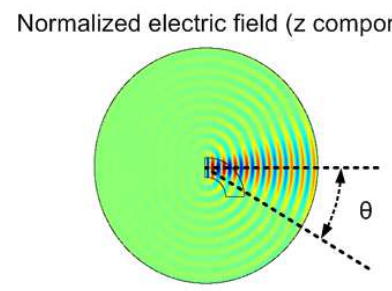

(a)

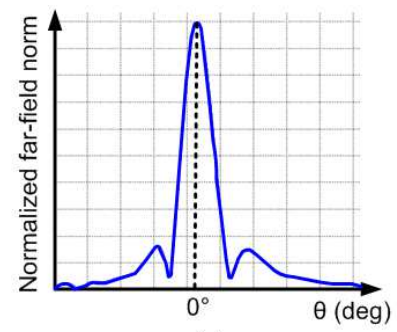

(c)

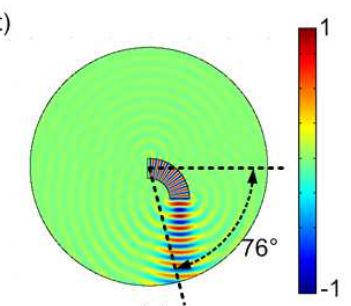

(b)

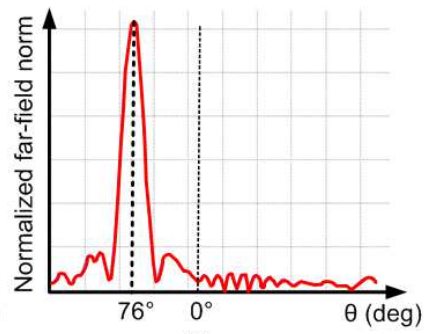

(d)
Figure 7: (a)-(b) Calculated emission of a plane current source above a limited metallic ground plane without and with the metamaterial structure. (c)-(d) Calculated normalized far field of the antenna without and with metamaterial. A $76^{\circ}$ rotation of the radiation is clearly observed.
For the physical prototype fabrication, we simplify the calculated material parameters through a parameter reduction procedure. We set a polarization of the electromagnetic field such that the magnetic field is along the z-direction. In this case, the relevant electromagnetic parameters are $\mu_{\mathrm{zz}}, \varepsilon_{\theta \theta}$ and $\varepsilon_{\text {rr. }}$. We maintain $\varepsilon_{\theta \theta}$ and $\mu_{\text {zz }}$ constant and the new set of coordinates is given by (6):

$$
\varepsilon_{r r}=\left(\frac{1}{b r}\right)^{2} \div 1.7 ; \varepsilon_{\theta \theta}=2.8 ; \mu_{z z}=1.7,
$$

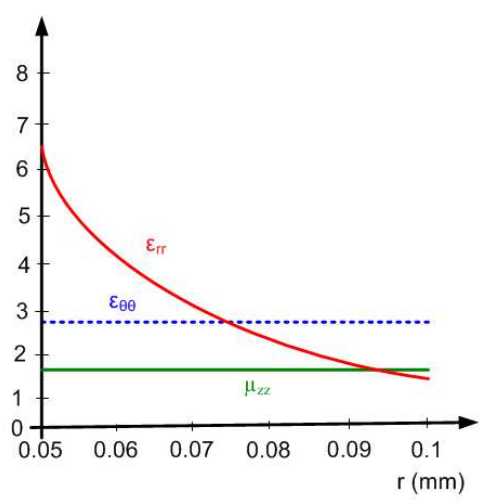

(a)

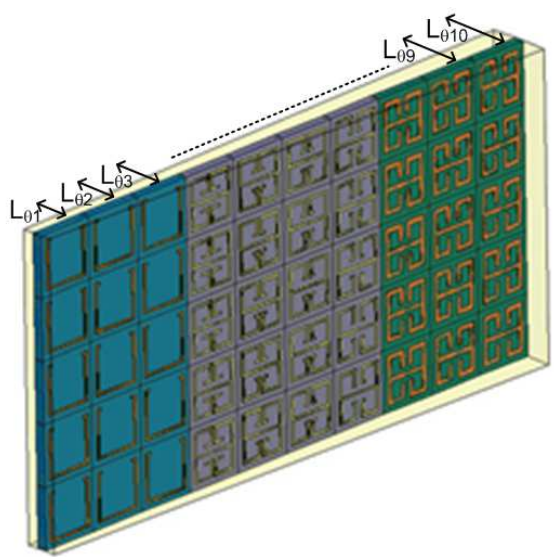

(b)
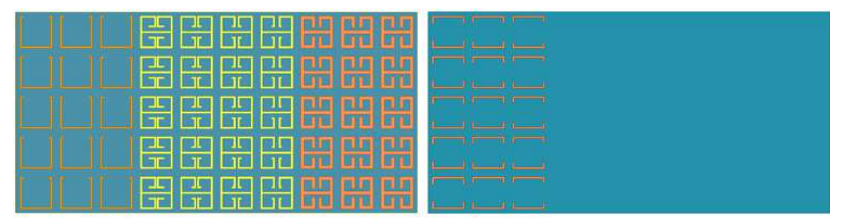

(c)

Figure 8: (a) Profile of the material parameters. (b) Single metamaterial layer composed of 10 unit cells providing the material parameters necessary for the coordinate transformation. (c) Front and rear view of the metamaterial cells.

Setting physical parameter $b=6$ allows an optimization of the material parameter $\varepsilon_{\mathrm{rr}}$. The profile of the different parameters is presented in Fig. 8(a). We consider fabricating the prototype composed of 30 identical layers where each layer is divided in 10 unit cells as illustrated by the single layer in Fig. 8(b). For the discretization of the material 
parameters, meta-atoms producing electric resonances are designed on the $0.787 \mathrm{~mm}$ thick low loss $(\tan \delta=0.0013)$ $\mathrm{RO} 3003^{\mathrm{TM}}$ dielectric substrate. We choose a rectangular unit cell with dimensions $5 \mathrm{~mm}$ for the resonators. We are able to obtain the desired $\varepsilon_{\mathrm{zz}}$ and $\mu_{\mathrm{yy}}$ by tuning the resonators' geometric parameters. The 10 cells presented in Fig. 8(c) are designed to constitute the discrete variation of $\varepsilon_{\text {rr }}$. Table I summarizes the corresponding electromagnetic parameters of the cells. The cells are composed of respectively SRRs and ELCs to secure $\mu_{\mathrm{zz}}$ and $\varepsilon_{\mathrm{rr}}$. $\varepsilon_{\theta \theta}$ is produced by a host medium, which is a commercially available resin.

Table 1: Electromagnetic parameters $\mu_{\mathrm{zz}}$, and $\varepsilon_{\mathrm{rr}}$ for the 10 cells of the metamaterial layers. The length $\mathrm{L}_{\theta}$ of each cell is given as a function of its position along the layer.

\begin{tabular}{ccccc}
\hline Layer & $\mathrm{r}_{\mathrm{i}}(\mathrm{mm})$ & $\mathrm{L}_{\theta \mathrm{i}}(\mathrm{mm})$ & $\mu_{\mathrm{zz}}$ & $\mathcal{E}_{\mathrm{rr}}$ \\
\hline 1 & 52.5 & 2.75 & 1.7 & 5.8 \\
2 & 57.5 & 3.01 & 1.7 & 4.842 \\
3 & 62.5 & 3.27 & 1.7 & 4.096 \\
4 & 67.5 & 3.53 & 1.7 & 3.504 \\
5 & 72.5 & 3.8 & 1.7 & 3.04 \\
6 & 77.5 & 4.06 & 1.7 & 2.664 \\
7 & 82.5 & 4.32 & 1.7 & 2.35 \\
8 & 87.5 & 4.58 & 1.7 & 2.09 \\
9 & 92.5 & 4.84 & 1.7 & 1.87 \\
10 & 97.5 & 5.1 & 1.7 & 1.68 \\
\hline
\end{tabular}

For numerical verifications of the proposed device performances, a microstrip patch antenna presenting a quasiomnidirectional radiation is used as the feeding source of the metamaterial antenna. This patch source is optimized for a $10 \mathrm{GHz}$ operation. A 3D simulation of the patch antenna and the layered metamaterial is performed using HFSS as illustrated in Fig. 9(a). Fig. 9(b) shows the calculated energy distribution in the middle plane of the layered metamaterial structure. We shall note that the latter structure firstly transforms the quasi-omnidirectional radiation of the patch source into a directive one and also maintains this highly directive emission after the $76^{\circ}$ rotation.

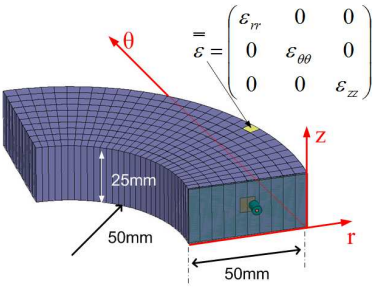

(a)

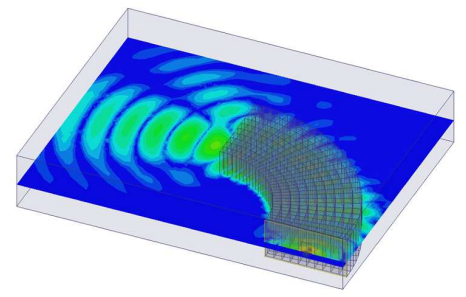

(b)
Figure 9: (a) Simulated design consisting of 30 metamaterial layers each composed of 10 cells. (b) Calculated energy distribution at $10 \mathrm{GHz}$.

To validate experimentally the azimuthal directive emission, the device shown in Fig. 10(a) is fabricated. A microstrip square patch antenna printed on a $1 \mathrm{~mm}$ thick epoxy dielectric substrate $\left(\varepsilon_{\mathrm{r}}=3.9\right.$ and $\left.\tan \delta=0.02\right)$ is used as radiating source. The metamaterial is a discrete structure composed of 10 different regions where permittivity and permeability vary according to Eq. (6) and to the values of
Table 1. The bulk metamaterial is assembled using 30 layers of $\mathrm{RO} 003^{\mathrm{TM}}$ dielectric boards on which subwavelength resonant structures are printed. The layers are mounted 1 by 1 in a molded matrix with a constant angle of $3^{\circ}$ between each. A commercially available liquid resin is then flowed into the mold. This resin constitutes the host medium and is an important design parameter which is closely linked to $\varepsilon_{\theta \theta}$. Its measured permittivity is close to 2.8 . The mold is removed after solidification of the resin.

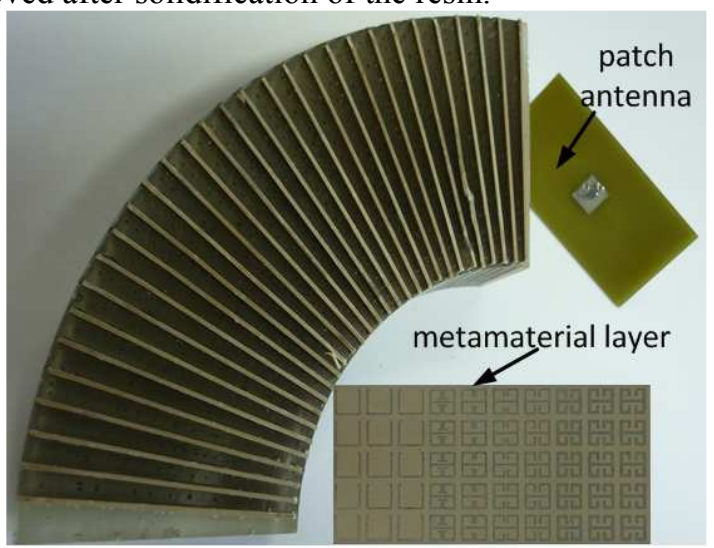

(a)

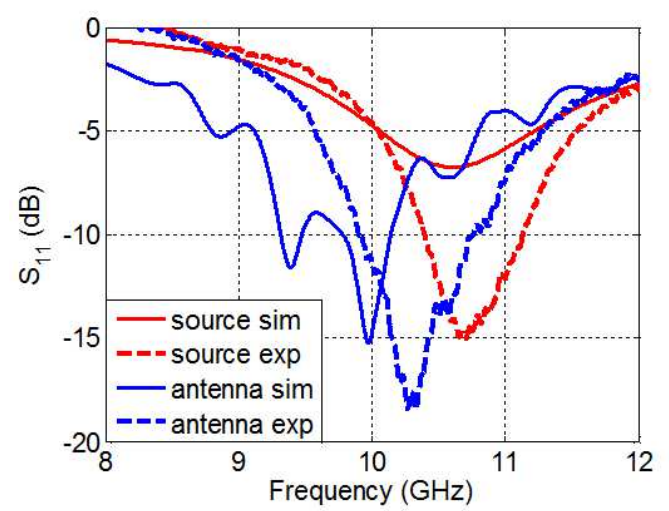

(b)

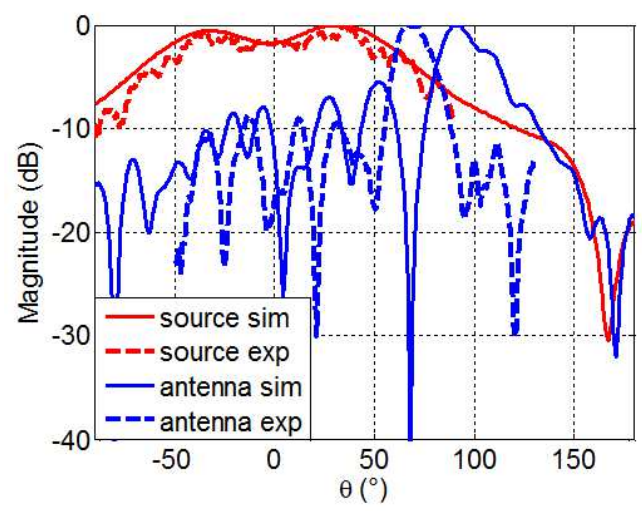

(c)

Figure 10: (a) Photography of the fabricated prototype. (b) Simulated and measured $S_{11}$ parameter of the patch source alone and the metamaterial antenna. (c) Far-field E-plane radiation patterns of the patch source alone and of the metamaterial antenna. 
$S_{11}$ parameter measurements are first performed on the fabricated prototype. The measured $S_{11}$ parameter of the metamaterial antenna is compared with the HFSS-simulated one in Fig. 10(b). A good agreement can be observed and return losses reaching $18 \mathrm{~dB}$ is observed experimentally at $10.3 \mathrm{GHz}$ compared to $15 \mathrm{~dB}$ calculated. This quantity is further compared with that of the feeding patch antenna alone. A better matching can be clearly observed for the metamaterial antenna. The E-plane far-field radiation pattern of the metamaterial antenna is measured in a fully anechoic chamber. Measurements are performed for computercontrolled elevation angle varying from $-90^{\circ}$ to $+90^{\circ}$. The measured far-field radiation pattern is presented for the metamaterial device (Fig. 10(c)). From the experiments, we can clearly observe the transformation of the omnidirectional far-field radiation of the patch antenna into a directive one which is further bent at an angle of $66^{\circ}$, which is consistent to the $76^{\circ}$ predicted by numerical simulations. The difference in bending angle is due to the fabrication tolerances of the meta-atoms providing the gradient radial permittivity and to the positioning of the patch source.

\section{Isotropic antenna}

Conversely to the previous sections, here we focus our attention on how coordinate transformation can be applied to transform directive emissions into isotropic ones. An intuitive schematic principle to illustrate the proposed method is presented in Fig. 11. Let us consider a source radiating in a circular space as shown in Fig. 11(a) and a circular region bounded by the blue circle around this source limits the radiation zone. The "space stretching" coordinate transformation consists in stretching exponentially the central zone of this delimited circular region represented by the red circle as illustrated in Fig. 11(b).

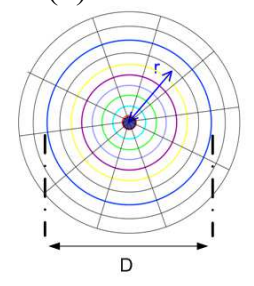

(a)

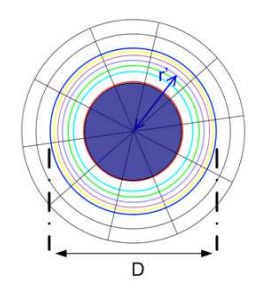

(b)

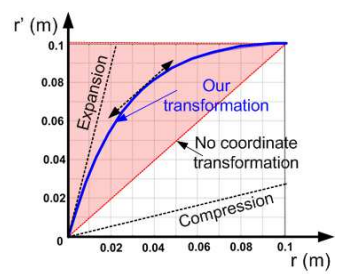

(c)
Figure 11: (a) initial space, (b) transformed space, (c) the blue curve shows the transformation rule made of an expansion followed by a compression.

The expansion procedure is further followed by a compression of the annular region formed between the red and blue circles so as to secure a good impedance matching with free space. Fig. 11(c) summarizes the exponential form of our coordinate transformation. The diameter of the transformed (generated metamaterial) circular medium is noted D.

Mathematically this transformation is expressed as [31]:

$$
\left\{\begin{array}{l}
r^{\prime}=\alpha\left(1-e^{q r}\right) \\
\theta^{\prime}=\theta \\
z^{\prime}=z
\end{array} \quad \text { with } \quad \alpha=\frac{\mathrm{D}}{2} \frac{1}{1-\mathrm{e}^{\frac{\mathrm{qD}}{2}}}\right.
$$

where $r^{\prime}, \theta^{\prime}$, and $z^{\prime}$ are the coordinates in the transformed cylindrical space, and $r, \theta$, and $z$ are those in the initial cylindrical space. In the initial space, we assume free space, with isotropic permittivity and permeability tensors $\varepsilon_{0}$ and $\mu_{0}$. Parameter $q$ (in $\mathrm{m}^{-1}$ ) appearing in Eq. (4) must be negative in order to secure the impedance matching condition. This parameter is an expansion factor which can be physically viewed as to what extent space is expanded. A high (negative) value of $q$ means a high expansion whereas a low (negative) value of $q$ means a nearly zero expansion.

Calculations lead to permeability and permittivity tensors given in the diagonal base by:

$=\left(\begin{array}{ccc}\psi_{r r} & 0 & 0 \\ 0 & \psi_{\theta \theta} & 0 \\ 0 & 0 & \psi_{z z}\end{array}\right)=\left(\begin{array}{ccc}\frac{q r\left(r^{\prime}-\alpha\right)}{r^{\prime}} & 0 & 0 \\ 0 & \frac{r^{\prime}}{q r\left(r^{\prime}-\alpha\right)} & 0 \\ 0 & 0 & \frac{r}{r^{\prime} q\left(r^{\prime}-\alpha\right)}\end{array}\right)$

with: $\mathrm{r}=\frac{\ln \left(1-\frac{\mathrm{r}^{\prime}}{\alpha}\right)}{q}$,

The components in the Cartesian coordinate system are calculated and are as follows:

$$
\left\{\begin{array}{l}
\psi_{x x}=\psi_{\mathrm{rr}} \cos ^{2}(\theta)+\psi_{\theta \theta} \sin ^{2}(\theta) \\
\psi_{x y}=\psi_{y x}=\left(\psi_{\mathrm{rr}}-\psi_{\theta \theta}\right) \sin (\theta) \cos (\theta) \\
\psi_{y y}=\psi_{\mathrm{rr}} \sin ^{2}(\theta)+\psi_{\theta \theta} \cos ^{2}(\theta)
\end{array}\right.
$$

By fixing the electric field directed along the $z$-axis and by adjusting the dispersion equation without changing propagation in the structure, the following reduced parameters can be obtained:

$$
\left\{\begin{array}{c}
\mu_{r r}=1 \\
\mu_{\theta \theta}=\left(\frac{r^{\prime}}{q r\left(r^{\prime}-\alpha\right)}\right)^{2}, \\
\varepsilon_{z z}=\left(\frac{r}{r^{\prime}}\right)^{2}
\end{array}\right.
$$

Fig. 12 presents simulations results of the source radiating in the initial circular space at an operating frequency of $4 \mathrm{GHz}$ for several values of $q$. The current direction of the source is supposed to be along the $z$-axis. Simulations are performed in a Transverse Electric (TE) mode with the electric field polarized along z-direction. The surface current source is considered to have a width of 10 
$\mathrm{cm}$, which is greater than the $7.5 \mathrm{~cm}$ wavelength at $4 \mathrm{GHz}$. Radiation boundary conditions are placed around the calculation domain in order to plot the radiation properties. Continuity of the field is assured in the interior boundaries. As stated previously and verified from the different electric field distribution patterns, a high negative value of $q$ leads to a quasi-perfect isotropic emission since the space expansion is higher. This phenomenon can be clearly observed in Fig. 12(d) for $q=-40 \mathrm{~m}^{-1}$.

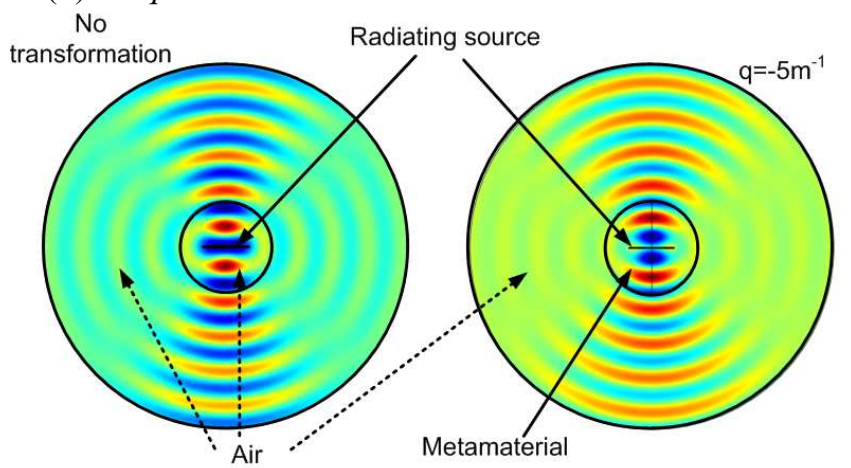

(a)

(b)

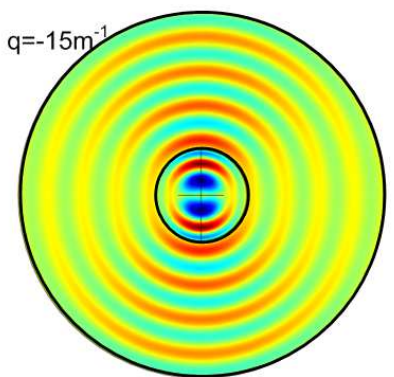

(c)

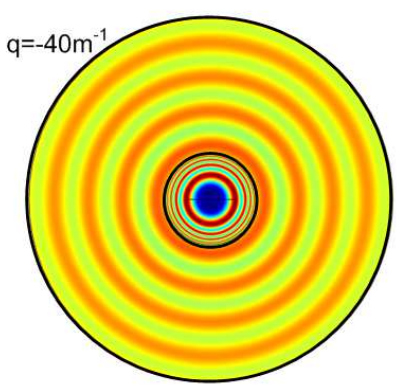

(d)
Figure 12: Simulated electric field distribution for a TE wave polarization at $4 \mathrm{GHz}$. (a) current plane source used as excitation for the transformation. The current direction is perpendicular to the plane of the figure; [(b)-(d)] verification of the transformation for different values of expansion factor $q$.

The calculated far-field patterns are shown in Fig. 13(a). The source alone produces a directive emission and when it is surrounded by the judiciously engineered coordinatetransformation based metamaterial, an isotropic emission is produced. Fig. 13(b) shows the influence of parameter $q$ on the space expansion in the coordinate transformation. As $q$ becomes highly negative, a greater space expansion is achieved.

\section{Conclusions}

To summarize, we have presented three types of antennas designed using coordinate transformation concept. The first antenna is a directive one achieved by transforming the omnidirectional radiation of a patch radiator into a directive one. Measurements performed on the fabricated prototype have shown good qualitative agreements with theory and simulations. The second antenna is an extension of the directive one, in the way that it also presents a steered beam. In this case, a rotational coordinate transformation has been elaborated to achieve the azimuthal emission.
Measurements have shown as much as $66^{\circ}$ beam steering. Finally, a space expansion coordinate transformation has been theoretically presented so as to transform a directive emission into an isotropic one.

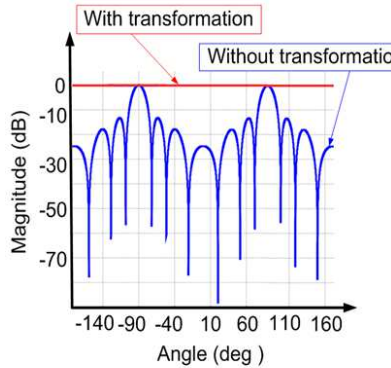

(a)

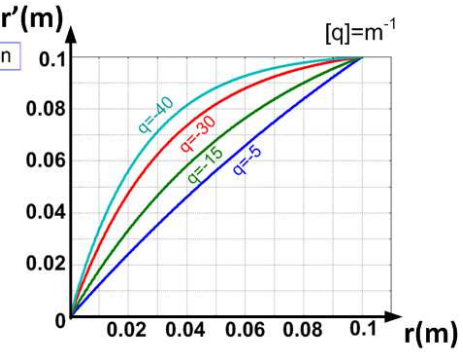

(b)
Figure 13: (a) Far field radiation pattern of the emission with $\left(q=-40 \mathrm{~m}^{-1}\right)$ and without transformation. (b) Influence of the expansion parameter $q$ on the proposed coordinate transformation. The emitted radiation is more and more isotropic as $q$ tends to high negative values.

\section{References}

[1] J. B. Pendry, D. Schurig, D. R. Smith, Controlling electromagnetic fields, Science 312(5781): 1780-1782, 2006.

[2] U. Leonhardt, Optical conformal mapping, Science 312(5781): 1777-1780, 2006.

[3] D. Schurig, J. J. Mock, B. J. Justice, S. A. Cummer, J. B. Pendry, A. F. Starr, D. R. Smith, Metamaterial electromagnetic cloak at microwave frequencies, Science 314(5801): 977-980, 2006.

[4] R. Liu, C. Ji, J. J. Mock, J. Y. Chin, T. J. Cui, D. R. Smith, Broadband ground-plane cloak, Science 323(5912): 366-369, 2009.

[5] B. Kanté, D. Germain, A. de Lustrac, Experimental demonstration of non-magnetic metamaterial cloak at microwave frequencies, Phys. Rev. B 80, 201104(R), 2009.

[6] W. Cai, U. K. Chettiar, A. V. Kildishev, V. M. Shalaev, Optical cloaking with metamaterials, Nat. Photon. 1(4): 224-227, 2007.

[7] J. Valentine, J. Li, T. Zentgraf, G. Bartal, X. Zhang, An optical cloak made of dielectrics, Nat. Mater. 8: 568$571,2009$.

[8] L. H. Gabrielli, J. Cardenas, C. B. Poitras, M. Lipson, Silicon nanostructure cloak operating at optical frequencies, Nat. Photon. 3(8): 461-463, 2009.

[9] M. Rahm, D. Schurig, D. A. Roberts, S. A. Cummer, D. R. Smith, J. B. Pendry, Design of electromagnetic cloaks and concentrators using form-invariant coordinate transformations of Maxwell's equations, Photon. Nanostruct.: Fundam. Appl. 6(1): 87-95, 2008.

[10] H. Chen, B. Hou, S. Chen, X. Ao, W. Wen, C. T. Chan, Design and experimental realization of a broadband transformation media field rotator at microwave frequencies, Phys. Rev. Lett. 102(18): 183903, 2009. 
[11]A. Greenleaf, Y. Kurylev, M. Lassas, G. Uhlmann, Electromagnetic wormholes and virtual magnetic monopoles from metamaterials, Phys. Rev. Lett. 99(18): 183901, 2007.

[12] M. Rahm, D. A. Roberts, J. B. Pendry, D. R. Smith, Transformation-optical design of adaptive beam bends and beam expanders, Opt. Express 16(15): 1155511567, 2008.

[13] M. Rahm, S. A. Cummer, D. Schurig, J. B. Pendry, D. R. Smith, Optical design of reflectionless complex media by finite embedded coordinate transformations, Phys. Rev. Lett. 100(6): 063903, 2008.

[14] L. Lin, W. Wang, J. Cui, C. Du, X. Luo, Design of electromagnetic refractor and phase transformer using coordinate transformation theory, Opt. Express 16(10): 6815-6821, 2008.

[15] J. Huangfu, S. Xi, F. Kong, J. Zhang, H. Chen, D. Wang, B.-I. Wu, L. Ran, J. A. Kong, Application of coordinate transformation in bent waveguide, J. Appl. Phys. 104(1): 014502, 2008.

[16]P.-H. Tichit, S. N. Burokur, A. de Lustrac, Waveguide taper engineering using coordinate transformation technology, Opt. Express 18(2): 767-772, 2010.

[17] W. X. Jiang, T. J. Cui, H. F. Ma, X. M. Yang, Q. Cheng, Layered high-gain lens antennas via discrete optical transformation, Appl. Phys. Lett. 93(22): 221906, 2008.

[18]Z. L. Mei, J. Bai, T. M. Niu, T. J. Cui, A Planar focusing antenna design using quasi-conformal mapping, PIER M 13: 261-273, 2010.

[19]Z. H. Jiang, M. D. Gregory, D. H. Werner, Experimental demonstration of a broadband transformation optics lens for highly directive multibeam emission, Phys. Rev. B 84(16): 165111, 2011.

[20]C. Garcia-Meca, A. Martinez, U. Leonhardt, Engineering antenna radiation patterns via quasiconformal mappings, Opt. Express 19(24): 2374323750, 2011.

[21] Y. G. Ma, C. K. Ong, T. Tyc, U. Leonhardt, An omnidirectional retroreflector based on the transmutation of dielectric singularities, Nat. Mater. 8: 639-642, 2009.

[22] T. Tyc, U. Leonhardt, "Transmutation of singularities in optical instruments," New J. Phys. 10(11): 115038, 2008.

[23]N. Kundtz, D. R. Smith, Extreme-angle broadband metamaterial lens, Nat. Mater. 9: 129-132, 2010.

[24]E. Lier, D. H. Werner, C. P. Scarborough, Q. Wu, J. A. Bossard, An octave-bandwidth negligible-loss radiofrequency metamaterial, Nat. Mater. 10(3): 216222, 2011.

[25] Y. Luo, J. Zhang, L. Ran, H. Chen, J. A. Kong, Controlling the Emission of Electromagnetic Source, PIERS Online 4(7): 795-800, 2008.
[26] J. Allen, N. Kundtz, D. A. Roberts, S. A. Cummer, D. R. Smith, Electromagnetic source transformations using superellipse equations, Appl. Phys. Lett. 94(19): 194101, 2009.

[27]B. I. Popa, J. Allen, S. A. Cummer, Conformal array design with transformation electromagnetics, Appl. Phys. Lett. 94(24): 244102, 2009.

[28]P.-H. Tichit, S. N. Burokur, A. de Lustrac, Ultradirective antenna via transformation optics, $J$. Appl. Phys. 105(10): 104912, 2009.

[29]P.-H. Tichit, S. N. Burokur, D. Germain, A. de Lustrac, Design and experimental demonstration of a highdirective emission with transformation optics, Phys. Rev. B 83(15): 155108, 2011.

[30] P.-H. Tichit, S. N. Burokur, D. Germain, A. de Lustrac, Coordinate transformation based ultra-directive emission, Elec. Lett. 47(10): 580-582, 2011.

[31]P.-H. Tichit, S. N. Burokur, A. de Lustrac, Transformation media producing quasi-perfect isotropic emission, Opt. Express 19(21): 20551-20556, 2011.

[32] J. B. Pendry, A. J. Holden, D. J. Robbins, W. J. Stewart, Magnetism from conductors and enhanced non-linear phenomena, IEEE Trans. Microwave Theory Tech. 47(11): 2075-2084, 1999.

[33]D. Schurig, J. J. Mock, D. R. Smith, Electric-fieldcoupled resonators for negative permittivity metamaterials, Appl. Phys. Lett. 88(4): 041109, 2006.

[34] A. M. Nicolson, G. F. Ross, Measurement of the intrinsic properties of materials by time-domain techniques, IEEE Trans. Instrum. Meas. 19(4): 377-382, 1970. 